\title{
RINGS WITH A JORDAN DERIVATION WHOSE IMAGE IS CONTAINED IN THE NUCLEI OR COMMUTATIVE CENTER
}

\author{
CHEN-TE YEN
}

\begin{abstract}
Let $R$ be a nonassociative ring, $N, L$ and $G$ the left nucleus, right nucleus and nucleus respectively. It is shown that if $R$ is a prime ring with a Jordan derivation $d$ such that $d(R) \subseteq G$, and $\left(d^{2}(R), R\right) \subseteq N$ or $\left(d^{2}(R), R\right) \subseteq L$ then either $R$ is associative or $2 d^{2}=0$. Moreover, if $(d(R), R)=0$ then either $R$ is associative and commutative, or $2 d=0$. We also prove that if $R$ is a prime ring with a derivation $d$ and there exists a fixed positive integer $n$ such that $d^{n}(R) \subseteq G$ and $\left(d^{n}(R), R\right)=0$ then $R$ is associative and $d^{n}=0$, or $R$ is associative and commutative, or $d^{2 n}=\left(\frac{(2 n) !}{n !}\right) d^{n}=0$. This partially generalize the results of [3]. We also obtain some results on prime rings with a derivation satisfying other hypotheses.
\end{abstract}

\section{Introduction}

Let $R$ be a nonassociative ring. We adopt the usual notations for associators and commutators : $(x, y, z)=(x y) z-x(y z)$ and $(x, y)=x y-y x$. We shall denote the left nucleus, middle nucleus, right nucleus and nucleus by $N, M, L$ and $G$ respectively. Thus $N, M, L$ and $G$ consists of all elements $n$ such that $(n, R, R)=0,(R, n, R)=0$, $(R, R, n)=0$ and $(n, R, R)=(R, n, R)=(R, R, n)=0$ respectively. An additive mapping $d$ from $R$ to $R$ is called a Jordan derivation if $d\left(x^{2}\right)=d(x) x+x d(x)$ holds for all $x$ in $R$. An additive mapping $d$ from $R$ to $R$ is called a derivation if $d(x y)=d(x) y+x d(y)$ holds for all $x, y$ in $R$. Obviously, every derivation is a Jordan derivation. The converse is in general not true. $R$ is called semiprime if the only ideal of $R$ which squares to zero is the zero ideal. $R$ is called prime if the product of any two nonzero ideals of $R$ is nonzero. Clearly, a prime ring is a semiprime ring. Herstein [1] proved that every Jordan derivation on a prime associative ring of characteristic not two is a derivation. Recently, Yen [3] showed the

Received May 25, 1993; revised December 4, 1995.

1991 Mathematics Subject Classification. Primary 17A36.

Key words and phrases. Nonassociative ring, nucleus, Jordan derivation, derivation, semiprime ring, prime ring. 
Theorem 1. If $R$ is a prime ring with a derivation $d$ such that $d(R) \subseteq N \cap L$ or $d(R) \subseteq N \cap M$ or $d(R) \subseteq M \cap L$, then either $R$ is associative or $d^{2}=2 d=0$.

In Theorem 1, if the derivation is replaced by the Jordan derivation then what can we say about $R$ and $d$ ? In fact, we prove that if $R$ is a prime ring with a Jordan derivation $d$ such that $d(R) \subseteq G$, and $\left(d^{2}(R), R\right) \subseteq N$ or $\left(d^{2}(R), R\right) \subseteq L$ then either $R$ is associative or $2 d^{2}=0$. Moreover, if $(d(R), R)=0$ then either $R$ is associative and commutative, or $2 d=0$. We also prove that if $R$ is a prime ring with a derivation $d$ and there exists a fixed positive integer $n$ such that $d^{n}(R) \subseteq G$ and $\left(d^{n}(R), R\right)=0$ then $R$ is associative and $d^{n}=0$, or $R$ is associative and commutative, or $d^{2 n}=\left(\frac{(2 n) !}{n !}\right) d^{n}=0$. This partially generalize Theorem 1 . We also obtain some results on prime rings with a derivation satisfying some hypotheses.

Assume that $R$ has a Jordan derivation $d$. Thus we have

$$
d\left(x^{2}\right)=d(x) x+x d(x) \text { for all } x \text { in } R .
$$

\section{Results}

Let $R$ be a nonassociative ring. In every ring one may verify the Teichmüller identity

$$
(w x, y, z)-(w, x y, z)+(w, x, y z)=w(x, y, z)+(w, x, y) z .
$$

Suppose that $n \in N$. Then with $w=n$ in (2) we obtain

$$
(n x, y, z)=n(x, y, z) \text { for all } n \text { in } N .
$$

Assume that $m \in L$. Then with $z=m$ in (2) we get

$$
(w, x, y m)=(w, x, y) m \text { for all } m \text { in } L .
$$

As consequences of (2), (3) and (4), we have that $N, M, L, N \cap M, M \cap L, N \cap L$ and $G$ are associative subrings of $R$.

We assume that $R$ has a Jordan derivation $d$ which satisfies

(*) $\quad d(R) \subseteq A$, where $A=N$, or $M$, or $L$.

Using (*) and a linearization of (1) gives

$$
d(x y+y x)=d(x) y+x d(y)+d(y) x+y d(x) \in A \text { for all } x, y, \text { in } R .
$$

Then with $x \in d(R)$ in (5), and using (*) and noting that $A$ is an associative subring of $R$, we get

$$
d^{2}(x) y+y d^{2}(x) \in A \text { for all } x, y \text { in } R .
$$


Definition. Let $I$ be the associator ideal of $R$. I consists of the smallest ideal which contains all associators.

Note that I may be characterized as all finite sums of associators and right (or left) multiples of associators, as a consequence of (2). Hence we have

$$
I=(R, R, R)+(R, R, R) R=(R, R, R)+R(R, R, R) .
$$

We assume that $R$ satisfies

$$
(* *) \quad\left(d^{2}(R), R\right) \subseteq B, \text { where } B=N, \text { or } L
$$

Note that if $B$ is a Lie ideal of $R$ i.e., $(B, R) \subseteq B$, and if $d^{2}(R) \subseteq B$, then we obtain $\left(d^{2}(R), R\right) \subseteq(B, R) \subseteq B$. Assume that $A=B=N$ or $L$. Using $(* *)$, we get $\left(d^{2}(x), y\right) \in$ $A$ for all $x, y$ in $R$. Combining this with (6) yields $2 d^{2}(x) y \in A$ and $2 y d^{2}(x) \in A$. Thus we have

$$
2 d^{2}(R) R \subseteq A \text { and } 2 R d^{2}(R) \subseteq A \text { if } A=B=N \text { or } L .
$$

Applying (8), (*), (3) and (4), and with $n \in 2 d^{2}(R)$ in (3), and with $m \in 2 d^{2}(R)$ in (4) respectively, we obtain

$$
2 d^{2}(R)(R, R, R)=0 \text { if } A=B=N \text { and } 2(R, R, R) d^{2}(R)=0 \text { if } A=B=L .
$$

Combining (9) with $(*)$ yields

$$
2 d^{2}(R)((R, R, R) R)=0 \text { if } A=B=N \text { and } 2(R(R, R, R)) d^{2}(R)=0 \text { if } A=B=L .
$$

Using (7), (9) and (10), we get

$$
2 d^{2}(R) \cdot I=0 \text { if } A=B=N \text { and } I \cdot 2 d^{2}(R)=0 \text { if } A=B=L .
$$

Lemma 1. If $R$ is a ring with a Jordan derivation d such that $d(R) \subseteq N \cap M$ and $\left(d^{2}(R), R\right) \subseteq N$ (resp. $d(R) \subseteq M \cap L$ and $\left.\left(d^{2}(R), R\right) \subseteq L\right)$, then $2 d^{2}(R) R \subseteq N \cap M$ and $2 R d^{2}(R) \subseteq N \cap M$ (resp. $2 d^{2}(R) R \subseteq M \cap L$ and $\left.2 R d^{2}(R) \subseteq M \cap L\right)$.

Proof. By symmetry, we only prove the lemma in case $d(R) \subseteq N \cap M$ and $\left(d^{2}(R), R\right) \subseteq N$. By (8), $2 d^{2}(R) R \subseteq N$. Using these, (6) and (2), for all $x, y, z, w \in R$ we obtain $0=2\left(d^{2}(x) y, z, w\right)=-2\left(y d^{2}(x), z, w\right)=-2\left(y, d^{2}(x) z, w\right)$. Thus $\left(y, 2 d^{2}(x) z, w\right)=$ 0 . Hence $2 d^{2}(R) R \subseteq M$. By $(6), 2 d^{2}(x) y+2 y d^{2}(x) \in N \cap M$. Since $2 d^{2}(x) y \in M$, this implies $2 y d^{2}(x) \in M$. By (8), $2 y d^{2}(x) \in N$, as desired.

Lemma 2. If $R$ is a ring with a Jordan derivation d such that $d(R) \subseteq G$, and $\left(d^{2}(R), R\right) \subseteq N$ or $\left(d^{2}(R), R\right) \subset L$, then the ideal $C$ of $R$ generated by $2 d^{2}(R)$ is $C=2\left\{d^{2}(R)+d^{2}(R) R+R d^{2}(R)+R \cdot d^{2}(R) R\right\}$. 
Proof. By symmetry, we only prove the lemma in case $d(R) \subseteq G$ and $\left(d^{2}(R), R\right) \subseteq$ $N$. Then by Lemma 1, we have $2 d^{2}(R) R \subseteq N \cap M$ and $2 R d^{2}(R) \subseteq N \cap M$. We see that $C$ is an additive subgroup of $(R,+)$. Thus we have

$$
2\left(R \cdot d^{2}(R) R\right) R=2 R\left(d^{2}(R) R \cdot R\right)=2 R \cdot d^{2}(R)\left(R^{2}\right) \subseteq 2 R \cdot d^{2}(R) R
$$

and

$$
\begin{aligned}
2 R\left(R \cdot d^{2}(R) R\right) & =2 R\left(R d^{2}(R) \cdot R\right)=2\left(R \cdot R d^{2}(R)\right) R \\
& =2\left(R^{2}\right) d^{2}(R) \cdot R \subseteq 2 R d^{2}(R) \cdot R=2 R \cdot d^{2}(R) R .
\end{aligned}
$$

Hence $C$ is an ideal of $R$.

Theorem 2. If $R$ is a prime ring with a Jordan derivation $d$ such that $d(R) \subseteq G$, and $\left(d^{2}(R), R\right) \subseteq N$ or $\left(d^{2}(R), R\right) \subseteq L$ then either $R$ is associative or $2 d^{2}(x)=4 d(x) d(x)=4 d(x) d(y)+4 d(y) d(x)=0$ for all $x, y$ in $R$.

Proof. By symmetry, we only prove the theorem in case $d(R) \subseteq G$ and $\left(d^{2}(R), R\right) \subseteq$ $N$. By $(11), 2 d^{2}(R) \cdot I=0$. Using Lemma 1 , we have $2 d^{2}(R) R \subseteq N \cap M$ and $2 R d^{2}(R) \subseteq$ $N \cap M$. Applying these and $d(R) \subseteq G$, we obtain that $2\left\{d^{2}(R)+d^{2}(R) R+R d^{2}(R)+\right.$ $\left.R \cdot d^{2}(R) R\right\} \cdot I=0$. If $I=0$, then $R$ is associative. Assume that $I \neq 0$.

Thus by Lemma 2 and the primeness of $R, C \cdot I=0$ implies $C=0$. Hence $2 d^{2}(R)=0$. Assume that $x, y \in R$. Using this, (1) and (5), we get

$$
0=2 d^{2}\left(x^{2}\right)=2 d(d(x) x+x d(x))=2 d^{2}(x) x+4 d(x) d(x)+2 x d^{2}(x)=4 d(x) d(x) .
$$

A linearization of this gives $4 d(x) d(y)+4 d(y) d(x)=0$, as desired.

Lemma 3. If $R$ is a ring and $(n, R)=0$, and $n \in N \cap M$ or $n \in M \cap L$ or $n \in N \cap L$ then $n \in G$.

Proof. We only prove the lemma in case $(n, R)=0$, and $n \in N \cap M$. Then for all $x, y$ in $R$, we have

$$
(x y) n=n(x y)=(n x) y=(x n) y=x(n y)=x(y n)
$$

as desired.

Theorem 3. If $R$ is a prime ring with a Jordan derivation $d$ such that $(d(R), R)=0$, and $d(R) \subseteq N \cap M$ or $d(R) \subseteq M \cap L$ or $d(R) \subseteq N \cap L$, then either $R$ is associative and commutative, or $2 d=0$.

Proof. By Lemma 3, $d(R) \subseteq G$. Assume that $R$ is associative. Since $(d(R), R)=0$, for all $x, y$ in $R$ we have $0=\left(d\left(x^{2}\right), y\right)=2(d(x) x, y)=2 d(x)(x, y)$. Thus $2 d(x) R(x, y)=$ 0 . By the primeness of $R$, this implies $2 d(x)=0$ or $(x, y)=0$ for all $x, y$ in $R$. It is well known that the additive group $(R,+)$ can not be the union of two proper subgroups. Hence we obtain $2 d(R)=0$ or $R$ is commutative. Assume that $R$ is not associative. 
Using Theorem 2, we have $(2 d(x))^{2}=0$ for all $x$ in $R$. Thus $2 d(x) R$ is an ideal of $R$ and $(2 d(x) R)^{2}=0$. By the semiprimeness of $R$, this implies $2 d(x) R=0$. Hence $2 d(R) R=0$. Thus we see that the ideal of $R$ generated by $2 d(R)$ is $2 d(R)$. By the primeness of $R$, $2 d(R) R=0$ implies $2 d(R)=0$, as desired.

Note that if $R$ is commutative then by (5), $2 d(x y)=2\{d(x) y+x d(y)\}$ for all $x, y$ in $R$. Combining this with Theorem 3 yields the

Corollary 1. If $R$ is a prime ring of characteristic not two with a Jordan derivation $d$ such that $(d(R), R)=0$, and $d(R) \subseteq N \cap M$ or $d(R) \subseteq M \cap L$ or $d(R) \subseteq N \cap L$, then $d$ is a derivation, and either $R$ is associative and commutative, or $d=0$.

In the course of the proofs of Theorems 2 and 3 , we obtain the

Corollary 2. If $R$ is a semiprime ring with a Jordan derivation $d$ such that $d(R) \subseteq G \cap I$ and $\left(d^{2}(R), R\right) \subseteq N$ or $\left(d^{2}(R), R\right) \subseteq L$, then $2 d^{2}=0$.

Corollary 3. If $R$ is a semiprime ring with a Jordan derivation d such that $(d(R), R)=0$ and $d(R) \subseteq G \cap I$, then $2 d=0$.

Corollary 4. If $R$ is a semiprime ring such that the Abelian group $(R,+)$ has no elements of order 2 and with a Jordan derivation d such that $(d(R), R)=0$ and $d(R) \subseteq G \cap I$, then $d=0$.

Recall that $R$ is a simple ring if $R$ is the only nonzero ideal of $R$. In [3], we proved that if $R$ is a simple ring with a derivation $d$ such that $d(R) \subseteq N \cap L$ then either $R$ is associative or $d^{2}=2 d=0$. For the prime ring case, we obtain the

Theorem 4. If $R$ is a prime ring with a derivation d such that $d(R) \subseteq N \cap M$ or $d(R) \subseteq M \cap L$ or $d(R) \subseteq N \cap L$, and there exists an ideal $T$ of $R$ such that $d(T)=0$, then either $T=0$ or $d=0$.

Proof. By symmetry, we only prove the theorem in case $d(R) \subseteq N \cap L$ and $d(T)=0$. For all $t \in T$ and $x \in R$, using $d(T)=0$ we have $0=d(t x)=d(t) x+$ $t d(x)=t d(x)$. Thus $T d(R)=0$. Because of $d(R) \subseteq N \cap L$, this implies $T(R d(R))=$ $(T R) d(R) \subseteq T d(R)=0$. Hence $T(d(R)+R d(R))=0$. Since $d$ is a derivation, we get $d(R)+d(R) R=d(R)+R d(R)$. By Lemma 1 of [3], or it is easy to see that $d(R)+R d(R)$ is an ideal of $R$. Thus by the primeness of $R, T(d(R)+R d(R))=0$ implies $T=0$ or $d(R)+R d(R)=0$, as desired.

Corollary 5. If $R$ is a prime ring with a derivation $d$ such that $d(I)=0$, and $d(R) \subseteq N \cap M$ or $d(R) \subseteq M \cap L$ or $d(R) \subseteq N \cap L$, then either $R$ is associative or $d=0$.

Theorem 5. If $R$ is a prime ring with a derivation $d$ and there exists an ideal $T$ of $R$ such that $d(T)=0$, and $T \subseteq N$ or $T \subseteq L$, then either $T=0$ or $d=0$. 
Proof. By symmetry, we only prove the theorem in case $d(T)=0$ and $T \subseteq N$. As in the proof of Theorem 4, we obtain that $T d(R)=0$, and we see that the ideal $E$ of $R$ generated by $d(R)$ is

$$
\begin{aligned}
E= & d(R)+d(R) R+d(R) R \cdot R+R \cdot d(R) R+(d(R) R \cdot R) R \\
& +R(d(R) R \cdot R)+(R \cdot d(R) R) R+R(R \cdot d(R) R)+\cdots .
\end{aligned}
$$

Because of $T \subseteq N$ and $T d(R)=0$, we have that

$$
\begin{gathered}
T(d(R) R \cdot R)=(T \cdot d(R) R) R=(T d(R) \cdot R) R=0, \\
T \cdot(d(R) R \cdot R) R=T(d(R) R \cdot R) \cdot R=0, \\
T(R \cdot d(R) R)=T R \cdot d(R) R \subseteq T \cdot d(R) R=T d(R) \cdot R=0
\end{gathered}
$$

and

$$
T(R \cdot(R \cdot d(R) R))=T R \cdot(R \cdot d(R) R) \subseteq T(R \cdot d(R) R)=0 .
$$

Thus by induction we can show that $T \cdot E=0$. By the primeness of $R$, this implies $T=0$ or $E=0$. Hence, either $T=0$ or $d=0$, as desired.

We have a very easy consequence of Theorem 5 .

Corollary 6. If $R$ is a prime associative ring and there exists an ideal $T$ of $R$ such that $d(T)=0$, then either $T=0$ or $d=0$.

Theorem 6. If $R$ is a prime ring with a derivation $d$ and there exists an ideal $T$ of $R$ such that $T \subseteq G$ and $d(T) \subseteq G$, then $R$ is associative, or $T=0$, or $d=0$.

Proof. By the hypotheses, for all $t \in T, x \in R$, we have $d(t x)=d(t) x+t d(x) \in G$ and so $d(t) x \in G$. Thus $d(T) R \subseteq G$. Hence by (3), we get $d(T)(R, R, R)=0$ and so $d(T)((R, R, R) R)=0$. Thus by $(7), d(T) \cdot I=0$. Using $d(T) \subseteq G$ and $d(T) R \subseteq G$, we see that the ideal $W$ of $R$ generated by $d(T)$ is

$$
W=d(T)+d(T) R+R d(T)+R \cdot d(T) R .
$$

Applying $d(T) \cdot I=0, d(T) \subseteq G$ and $d(T) R \subseteq G$, we obtain $W \cdot I=0$. By the primeness of $R$, this implies $W=0$ or $I=0$. If $I=0$, then $R$ is associative. Assume that $W=0$. Then $d(T)=0$. By Theorem 5, we have that $T=0$ or $d=0$. This completes the proof of Theorem 6.

Proposition. If $R$ is a ring with a derivation $d$, then the associative subrings $N, M$ and $L$ of $R$ are invariant under $d$, i.e., $d(A) \subseteq A$, where $A=N$ or $M$ or $L$.

Proof. Assume that $m \in M, x, y \in R$. Then by the definition of $d$, we obtain

$$
\begin{aligned}
(x, d(m), y) & =(x d(m)) y-x(d(m) y)=(d(x m)-d(x) m) y-x(d(m y)-m d(y)) \\
& =d(x m) y-d(x)(m y)-x d(m y)+(x m) d(y)=d((x m) y)-d(x(m y)) \\
& =d((x, m, y))=d(0)=0 .
\end{aligned}
$$


Thus $d(m) \in M$. Hence $d(M) \subseteq M$. The proofs of other cases are similar.

Theorem 7. If $R$ is a prime ring with a derivation $d$ and there exists a fixed positive integer $n$ such that $d^{n}(R) \subseteq G$ and $\left(d^{n}(R), R\right)=0$, then $R$ is associative and $d^{n}=0$, or $R$ is associative and commutative, or $d^{2 n}=\left(\frac{(2 n) !}{n !}\right) d^{n}=0$.

Proof. Note that $d^{2 n}(R) \subseteq d^{2 n-1}(R) \subseteq \cdots \subseteq d^{n+2}(R) \subseteq d^{n+1}(R) \subseteq d^{n}(R) \subseteq G$, or by the Proposition we have $d^{i}(R) \subseteq G$ for all integers $i \geq n$. Since $\left(d^{n}(R), R\right)=0$, we obtain $\left(d^{i}(R), R\right)=0, i \geq n$. Assume that $x, y, z \in R$. Using $d^{n}(R) \subseteq G$, we get $d^{n}\left(d^{n}(x) d^{n-1}(y)\right) \in G$. Expanding this by Leibniz's formula, and applying $d^{i}(R) \subseteq G$, $i \geq n$ and noting that $G$ is an associative subring of $R$, we have $d^{2 n}(x) d^{n-1}(y) \in G$. Thus $d^{2 n}(R) d^{n-1}(R) \subseteq G$. Using this and $\left(d^{i}(R), R\right)=0, i \geq n$, and argue as above, we get $d^{2 n}(x) d^{n-1}\left(d^{n}(y) d^{n-2}(z)\right) \in G$ and so $d^{2 n}(x)\left(d^{2 n-1}(y) d^{n-2}(z)\right) \in G$. Hence $\left(d^{2 n}(R) d^{2 n-1}(R)\right) d^{n-2}(R) \subseteq G$. Continuing in this manner, and applying $d^{i}(R) \subseteq G$ and $\left(d^{i}(R), R\right)=0, \quad i \geq n$, we finally obtain

$$
\left(d^{2 n}(R) d^{2 n-1}(R) \cdots d^{n+2}(R)\right) d(R) \subseteq G .
$$

Argue as above, we have $\left(d^{2 n}(R) d^{2 n-1}(R) \cdots d^{n+2}(R)\right) d\left(d^{n}(x) y\right) \subseteq G$ and so $\left(d^{2 n}(R) d^{2 n-1}(R) \cdots d^{n+2}(R)\right) d^{n+1}(x) y \subseteq G$. Therefore we get

$$
\left(d^{2 n}(R) d^{2 n-1}(R) \cdots d^{n+1}(R)\right) R \subseteq G .
$$

Using $d^{i}(R) \subseteq G, i \geq n$, we have

$$
\left(d^{2 n}(R) d^{2 n-1}(R) \cdots d^{n+1}(R)\right) \subseteq G .
$$

By (3), combining (12) with (13) yields

$$
\left(d^{2 n}(R) d^{2 n-1}(R) \cdots d^{n+1}(R)\right)(R, R, R)=0 .
$$

Using (13) and (14), we get

$$
\left(d^{2 n}(R) d^{2 n-1}(R) \cdots d^{n+1}(R)\right) \cdot(R, R, R) R=0 .
$$

By (7), combining (14) with (15) yields

$$
\left(d^{2 n}(R) d^{2 n-1}(R) \cdots d^{n+1}(R)\right) \cdot I=0 .
$$

Applying (13), $\left(d^{i}(R), R\right)=0$ and $d^{i}(R) \subseteq G, i \geq n$, we see that the ideal $U$ of $R$ generated by $\left(d^{2 n}(R) d^{2 n-1}(R) \cdots d^{n+1}(R)\right)$ is

$$
U=\left(d^{2 n}(R) d^{2 n-1}(R) \cdots d^{n+1}(R)\right)+\left(d^{2 n}(R) d^{2 n-1}(R) \cdots d^{n+1}(R)\right) R .
$$

Using (13), (16) and (17), we obtain $U \cdot I=0$. By the primeness of $R, U=0$ or $I=0$. If $I=0$, then $R$ is associative. By Theorem 1 of [2], either $d^{n}=0$ or $R$ is commutative. 
Assume that $U=0$. Thus we have

$$
d^{2 n}(R) d^{2 n-1}(R) \cdots d^{n+2}(R) d^{n+1}(R)=0 .
$$

As above, we see that the ideal $V$ of $R$ generated by $\left(d^{2 n}(R) d^{2 n-1}(R) \cdots d^{n+2}(R)\right)$ is

$$
V=\left(d^{2 n}(R) d^{2 n-1}(R) \cdots d^{n+2}(R)\right)+\left(d^{2 n}(R) d^{2 n-1}(R) \cdots d^{n+2}(R)\right) R .
$$

Applying $d^{i}(R) \subseteq G$ and $\left(d^{i}(R), R\right)=0, i \geq n,(18)$ and (19), we get $V^{2}=0$. By the semiprimeness of $R$, this implies $V=0$. Hence we obtain

$$
d^{2 n}(R) d^{2 n-1}(R) \cdots d^{n+2}(R)=0 .
$$

Continuing in this way, we can finally show that $d^{2 n}(R)=0$. Thus we get $0=$ $d^{2 n}\left(d^{2 n-2}(x) y\right)=(2 n) d^{2 n-1}(x) d^{2 n-1}(y)$. Using this, $\left(d^{i}(R), R\right)=0$ and $d^{i}(R) \subseteq G$, $i \geq n$, we have that $(2 n) d^{2 n-1}(x) R$ is an ideal of $R$ and $\left((2 n) d^{2 n-1}(x) R\right)^{2}=0$. By the semiprimeness of $R$, this implies $(2 n) d^{2 n-1}(x) R=0$. Hence $(2 n) d^{2 n-1}(R) R=0$. Thus the ideal of $R$ generated by $(2 n) d^{2 n-1}(R)$ is $(2 n) d^{2 n-1}(R)$. Therefore $(2 n) d^{2 n-1}(R)=0$. Continuing in this manner, and applying $d^{i}(R) \subseteq G$ and $\left(d^{i}(R), R\right)=0, i \geq n$, we finally obtain $(2 n)(2 n-1) \cdots(2 n-(n-1)) d^{n}(R)=0$. Thus $\left(\frac{(2 n) !}{n !}\right) d^{n}=0$. This completes the proof of Theorem 7 .

Corollary 7. If $R$ is a prime ring with a derivation $d$ and there exists a fixed positive integer $n$ such that $d^{n}(R) \subseteq G$ and $\left(d^{n}(R), R\right)=0$, and char $R$ does not divide $\left(\frac{(2 n) !}{n !}\right)$ then either $R$ is associative and commutative, or $d^{n}=0$.

Corollary 8. If $R$ is a semiprime ring with a derivation $d$ and there exists a fixed positive integer $n$ such that $d^{n}(R) \subseteq G \cap I$ and $\left(d^{n}(R), R\right)=0$, then $d^{2 n}=$ $\left(\frac{(2 n) !}{n !}\right) d^{n}=0$.

Theorem 7 partially generalizes Theorem 1 .

In [4], we extended Theorem 7 to $s$-derivation $d$ with $s d=d s$.

\section{References}

[1] I. N. Herstein, "Jordan derivations of prime rings," Proc. Amer. Math. Soc., 8(1957), 1104-1110.

[2] P. H. Lee and T. K. Lee, "Note on nilpotent derivations," Proc. Amer. Math. Soc., 98(1986), 31-32.

[3] C. T. Yen, "Rings with a derivation whose image is contained in the nuclei," Tamkang J. Math., 25(1994), 301-307.

[4] C. T. Yen, Nonassociative rings with skew derivations and rings with associators in the nuclei, Ph.D thesis, Taiwan University, 1995. 